\title{
Flexible Mass Spring Method for Modelling Soft Tissue Deformation
}

\author{
M.N. Omar ${ }^{*}$ and Y. Zhong ${ }^{2}$ \\ ${ }^{I}$ Faculty of Mechanical and Automotive Engineering Technology, Universiti Malaysia Pahang, 26600, Pekan, \\ Pahang, Malaysia \\ ${ }^{2}$ School of Engineering, RMIT University, 3083 Bundoora, Australia \\ *Email:nadzeri@ump.edu.my
}

\begin{abstract}
Soft tissues in general display two phases of deformation, linear during small deformation and nonlinear during large deformation. Researchers have been facing difficulty to model the soft tissue deformation mainly due to the two phases of deformation. Simplification often the solution in which either linear or nonlinear part is considered. On top of that, the nonlinearity of the deformation cannot be simply described by polynomial or exponential functions which have increased the complexity of the simulation process. This study explores an alternative simulation approach from the standpoint of Mass Spring Method (MSM). The proposed MSM model is developed using conical spring methodology which allows the MSM model to have different stiffnesses at different displacements during deformation. The stiffness variation creates flexibility in the MSM model to simulate any linear and nonlinear behaviors. This paper also analyzed the influence of several conical spring parameters on overall deformation. The experimental findings demonstrate that the proposed model produces deformations that are consistent with real and phantom soft tissue deformations. After the parameters are optimized, the average relative error is less than $5 \%$.
\end{abstract}

Indexed Terms- Soft tissue deformation, nonlinear MSM, conical spring methodology

\section{INTRODUCTION}

The most common methods for modeling soft tissue deformation are the Finite Element Method (FEM) and the Mass-Spring Method (MSM). The first approach is based on continuum mechanics, which governs elastic behavior in a continuous medium made up of linked volumes. FEM-based implementations are difficult. Although it depicts precise and realistic performance, FEM models are extremely detailed, intricate, and computationally demanding. The second approach, on the other hand, considers elastic behavior on a discrete basis. It divides a model into separate mass points linked together by springs. Traditional MSM models, in general, are controlled by significantly simpler mathematics than the FEM model. As a result, it provides simplicity and real-time interaction.

Numerous works have been published in the literature that aim on improving the MSM model in terms of modeling nonlinear deformation. Cooper and Maddock [5] presented one of the early nonlinear MSM models. They used a quadratic function to characterize the nonlinear behavior of soft tissues. The displacement function of a nonlinear spring was developed using the quadratic function. The method's primary disadvantages were that it was only applicable to two-dimensional objects and that no validation data was provided. Teschner et al. [6] went on to characterize the nonlinear behavior of their MSM model by varying the stiffness of the spring. Teschner et al. employed a quadratic function on the spring stiffness rather than a quadratic function on the displacement function, as Copper and Maddock did. They provided three stiffness functions for the user to choose from: linear, small nonlinear, and large nonlinear. Their methodology, however, is only viable for stiffness coefficients that converge to linear during small deformation. Furthermore, Luo and Xiao [7] used Duffing's equation to characterize the nonlinearity. It is a second-order nonlinear differential equation. Their investigation was successful in simulating nonlinearity, but no validation procedure was reported. Cui et al. [8] used the Duffing's 
equation as well, however, the approach for evaluating global and local deformation differed between these two studies.

San-Vicente et al. [9] reported the following method. The nonlinear response was accomplished in the study by combining linear springs in a cubical configuration. San-Vicente et al. employed three spring configurations: edge spring, diagonal spring, and internal diagonal spring. All springs in each configuration have a unique stiffness constant used to regulate the model's nonlinearity. Following that, Shah et al. [10] refined the earlier study by employing two rather than three configurations. Only the edge and diagonal spring configurations were employed by Shah et al. In addition, for stability, a cubic spring constant was incorporated into the internal force equation. Although both techniques were effective in replicating nonlinearity, the optimization process is still essential and time-consuming, especially when trying to determine the best settings for numerous distinct springs.

Another approach employed in the MSM model that can produce a nonlinear response is the load propagation method. This approach was employed in various tests by Choi et al. $[11,12]$. They developed a deformable model based on sequential force propagation. The number of springs engaged in deformation increased in proportion to the depth of the displacement. As a result, the resistance force was increased, resulting in a nonlinear reaction. Although the methodology may mimic nonlinearity, the nonlinearity of the model is not smooth and appears to be a mixture of two linear functions.

The piecewise technique is another way for modeling nonlinearity. The deformation behavior is broken down into sections, each with its formulation. Basafa et al. [13], for example, divided deformation behavior into two sections. The first section is linear and is controlled by a linear function, whereas the second section is nonlinear and is controlled by a nonlinear function. There are, on the other hand, piecewise approaches that employ just linear functions to imitate nonlinearity. This method, however, requires the use of more than two linear functions to provide a smooth nonlinear response. It is evidence by contrasting the work of Keeve et al. [14] and Garcia et al. [15]. Keeve et al. utilized two linear functions to illustrate nonlinear behavior, but Garcia et al. employed more than two linear functions. While more linear functions provide higher accuracy, it requires more processing time.

This research investigates an MSM modeling approach based on conical spring methodology. The conical spring's load and displacement relationship is established by discretizing the spring into several elementary parts. Each elementary part has a unique radius towards the spring's normal axis due to the conical shape. As a result, each elementary part has a distinct load and displacement relationship, which induces stiffness variation. The conical spring has the advantage of allowing stiffness variation to be controlled by adjusting the conical parameters. The parameters influence the rate of stiffness variation. Nonlinearity is reduced in a conical spring with a low rate of stiffness variation. In the meanwhile, increasing the rate of stiffness variation can lead to higher nonlinearity. This research builds on previous work [Omar] by focusing on the conical spring settings that control the rate of stiffness variation to replicate any deformation behavior.

\section{METHODOLOGY}

\subsection{Fundamental of cylindrical spring stiffness.}

The fundamental determination of the cylindrical spring force is used in conical spring methodology. Wahl [16] discretized a cylindrical spring into multiple elementary parts to compute deflection, and the resulting axial deflection $\delta$ when the cylindrical spring is subjected to a load can be determined using the following equation

$P=\frac{G d^{4}}{64 r^{3} n_{a}} \delta$ 

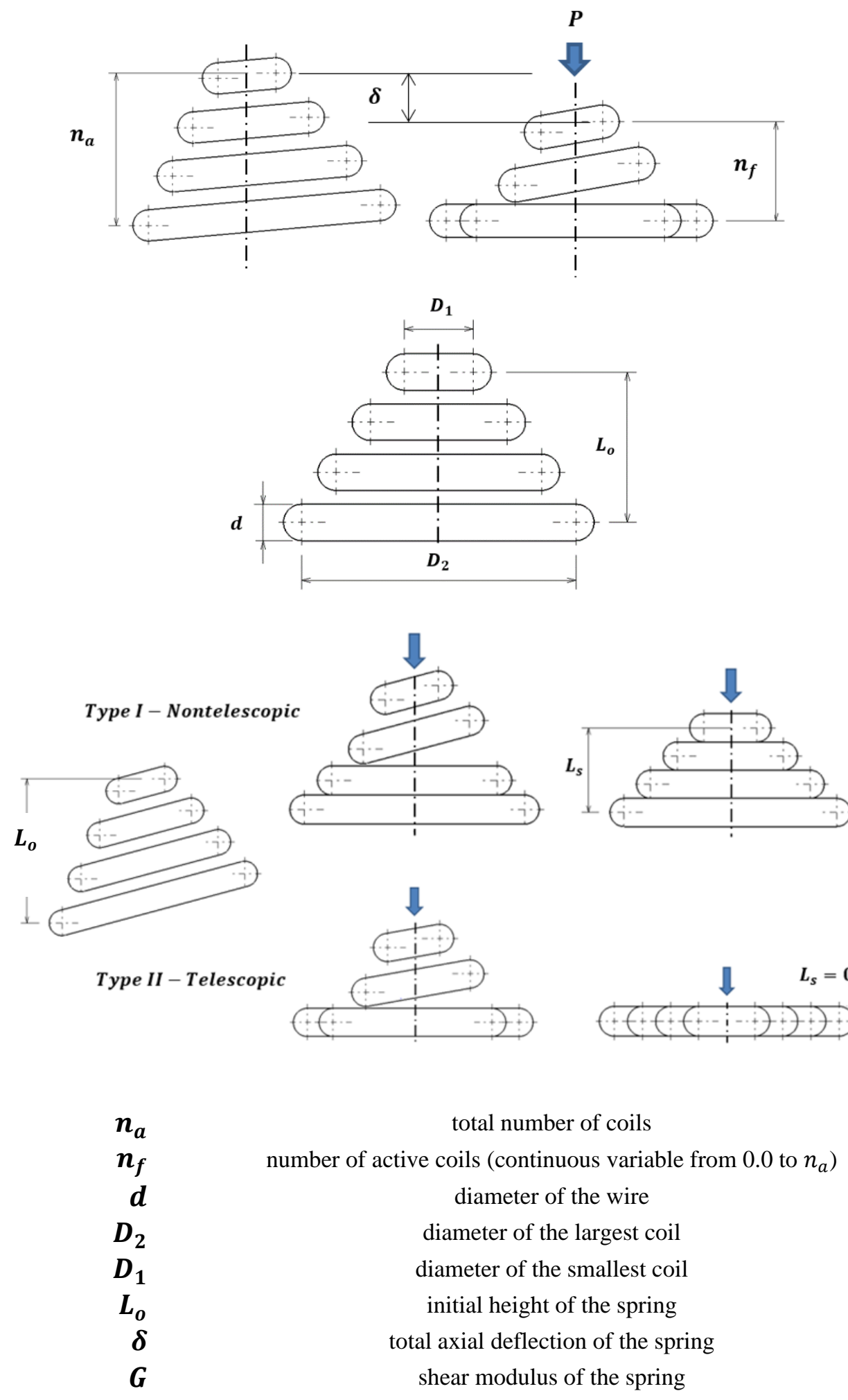

Figure 1: Parameterisation of conical spring. 
where $P$ denotes normal load, $r$ denotes mean radius of the spring coil, $d$ denotes diameter of the spring wire, $n_{a}$ denotes number of coils and $G$ denotes shear modulus. Regarding Hooke's law, spring stiffness $K$ can be obtained from equation (1) as

$K=\frac{G d^{4}}{64 r^{3} n_{a}}$

When all parameters of the cylindrical spring are constant, the relationship between load and axial deflection is linear, and the spring stiffness is constant, according to equation (2). Meanwhile, by varying the radius of the spring, different spring stiffness values can be obtained.

\subsection{Types of conical spring}

According to Paredes and Rodriguez [17], a conical spring is characterized using six design parameters, as shown in Figure. 1. This research solely looks at the derivation of a conical spring with a constant pitch value. The pitch value represents the distance between the coils of the conical spring. The pitch value is not specified throughout this study, but it is represented by the initial length and the total number of coils in the conical spring.

Conical springs are classified into two types based on their height when fully compressed Ls. The height can be determined using the following equation

$L_{s}=\left\{\max \left[0,\left(n_{a} d\right)^{2}-\left(r_{2}-r_{1}\right)^{2}\right]\right\}^{1 / 2}$

The spring coils for the first type of conical spring, known as non-telescopic, are piled on top of one another. As a result, the height at fully compressed represents the total of all coil heights. Meanwhile, the height at the completely compressed condition for the second type of conical spring, denoted as telescopic, on the other hand, is equal to zero since all coils are compressed to the ground (see Figure. 1). Analytically, the following relationships can be used to distinguish the type of conical spring

$$
\begin{array}{ll}
\left(r_{2}-r_{1}\right)<n_{a} d & \text { Type I } \\
\left(r_{2}-r_{1}\right) \geq n_{a} d & \text { Type II }
\end{array}
$$

where $r_{1}$ and $r_{2}$ are the radiuses of the smallest and the largest coil respectively.

\subsection{Formulation of conical spring}

The radius of the active coil $r(n)$ of a conical spring with a constant pitch size may be calculated using

$r(n)=\left[r_{1}+\left(r_{2}-r_{1}\right) \frac{n}{n_{a}}\right]$

where $n$ is the number of active coils in the spring coil sequence, which is a continuous variable ranging from 0.0 to the total number of coils $n_{a}$.

The elementary deflection $\delta_{e}$ can be determined for a given radius $r(n)$ that corresponds to the position of the current active coil as

$\delta_{e(n)}=\frac{64 P r^{3}}{G d^{4}} d n$

Furthermore, as seen in Figure. 1, the maximum elementary deflection $\delta_{e \text { max }}$ for any number of active coils $n$ can be evaluated using

$\delta_{\text {emax }}=\frac{L_{o}-L_{S}}{n_{a}} d n$ 
where $L_{o}$ denotes initial height of the spring.

The total spring deflection can be calculated as the sum of the elementary coil deflection $\delta_{e}$ (before fully compressed) and the fully compressed coil deflection $\delta_{e \text { max }}$ noted as

$\delta=\delta_{e}+\delta_{e \max }$

$\delta=\int_{0}^{n} \delta_{e(n)}+\int_{n}^{n_{a}} \delta_{e \max }$

By solving the integration, the total spring deflection at any axial load value can be obtained by

$\delta(P)=\frac{2 P\left(D_{1}\right)^{4} n_{a}}{G d^{4}\left(D_{2}-D_{1}\right)}\left[\left[1+\left(\frac{D_{2}}{D_{1}}-1\right) \frac{n}{n_{a}}\right]^{4}-1\right]+\left(L_{o}-L_{S}\right)\left(1-\frac{n}{n_{a}}\right)$

Eliminating the term $n$ with the diameter of the smallest and biggest coils as derived in equation (6), the inverse equation for equation (11) of load $P$ in terms of deflection $\delta$ can be calculated using the following equation

$P(\delta)=\left(\frac{A_{1}}{2}\right)^{\frac{3}{2}}\left[1-\left(1-2\left[1-\left(1+\frac{A_{2}}{A_{1}{ }^{2}}\right)^{\frac{1}{2}}\right]\right)^{\frac{1}{2}}\right]^{3}$

where $A_{1}$ to $A_{7}$ are given as

$A_{1}=A_{3}-\frac{A_{2}}{3 A_{3}}$

$A_{2}=-\frac{A_{6}}{A_{5}}$

$A_{3}=\left[\frac{A_{4}}{16}+\left[\left(\frac{A_{4}}{16}\right)^{2}+\left(\frac{A_{2}}{3}\right)^{3}\right]^{\frac{1}{2}}\right]^{\frac{1}{3}}$

$A_{4}=\left[\frac{\left(A_{7}+\delta\right)}{A_{5}}\right]^{2}$

$A_{5}=-\frac{2 D_{1}^{4} n_{a}}{G d^{4}\left(D_{2}-D_{1}\right)}$

$A_{6}=-\frac{3}{8\left(D_{2}-D_{1}\right)}\left[\frac{G d^{4}\left(L_{o}-L_{S}\right)}{n_{a}}\right]^{\frac{1}{3}}$

$A_{7}=\left(L_{o}-L_{S}\right) \frac{D_{2}}{\left(D_{2}-D_{1}\right)}$

The terms $A_{2}, A_{5}, A_{6}$ and $A_{7}$ can be precomputed from the conical spring parameters, while the total deflection $\delta$ equals the displacement of the conical spring.

\subsection{Model development}

A cubical object is discretised into $\eta$ mass points $\boldsymbol{u}_{i}$ with mass $m_{i}, i=1, \ldots, \eta$. The mass points are connected to each other via conical spring in the shape of hexahedral element (see Figure. 2 and Figure. 3 ). When a mass point moves as a result of an external force, the enforced displacement is transmitted 
to neighbouring mass points via the springs. The model deforms as a result of this. The model state at time $t$ is determined by the position $\boldsymbol{u}_{i}$ and velocity $\dot{\boldsymbol{u}}_{i}$ of every mass point.

The dynamic equilibrium of the MSM model is described using Newton's second law of motion. The induced external load is balanced by internal forces, which include the damping force, which is responsible for producing resistance due to mass velocity, and the spring force, which describes the resistance experienced by the spring. The dynamic equilibrium of each affected mass point can be expressed as

$m_{i} \boldsymbol{a}_{i}+\boldsymbol{F}_{d i}+\boldsymbol{F}_{k i}=\boldsymbol{F}_{\text {ext } \_i}$

where $m_{i}$ denotes mass at point $i, \boldsymbol{a}_{i}$ denotes the acceleration at point $i, \boldsymbol{F}_{d i}$ denotes the damping force at point $i, \boldsymbol{F}_{k i}$ denotes the spring force at point $i$, and $\boldsymbol{F}_{\text {ext }} i$ denotes the external force applied on point $i$.

Equation (20) can be expressed in terms of displacement as

$m_{i} \ddot{\boldsymbol{u}}_{l}+d_{i j} \dot{\boldsymbol{u}}_{i}+\boldsymbol{F}_{k i}\left(\boldsymbol{u}_{i}\right)=\boldsymbol{F}_{\text {ext } \_i}$

where $d_{i j}$ denotes damping constant between points $i$ and $\mathrm{j}$. The terms $\boldsymbol{u}_{i}, \dot{\boldsymbol{u}}_{i}$ and $\ddot{\boldsymbol{u}}_{\boldsymbol{\imath}}$ denote the displacement, velocity and acceleration of point $i$ respectively. The spring force at point $i$, is represented by the conical spring force $\boldsymbol{F}\left(\boldsymbol{u}_{i}\right)$, as described in equation (12).

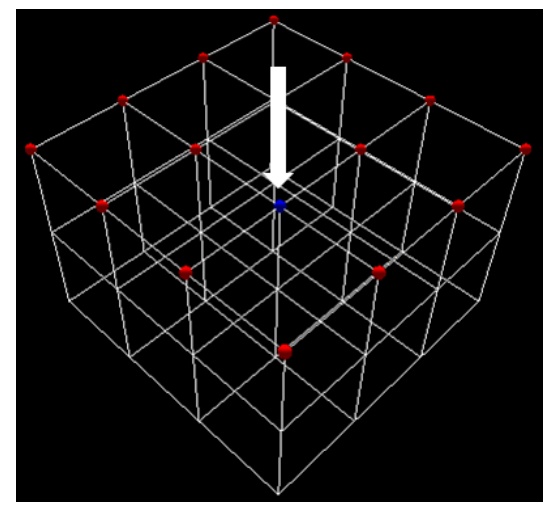

Figure 2: Conical spring model structure.
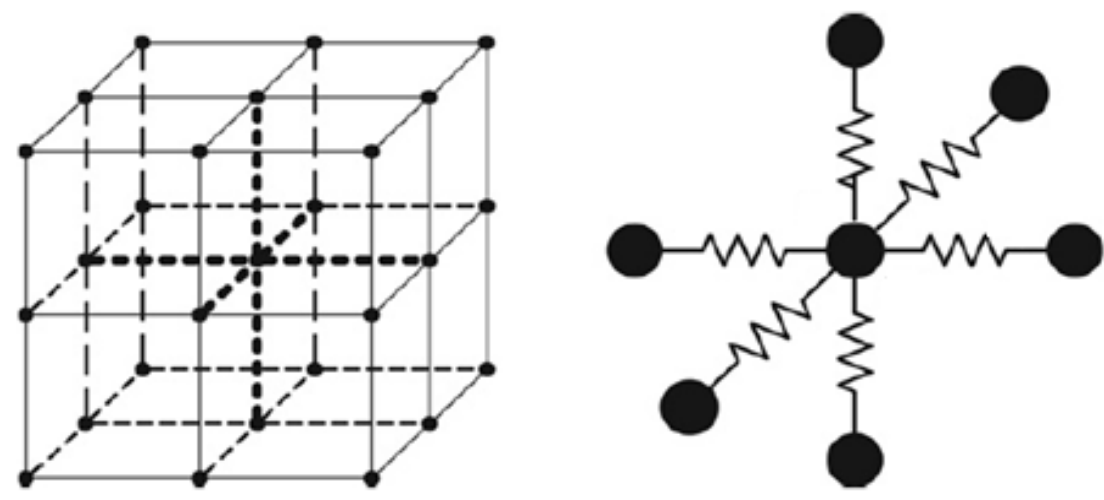

Figure 3: Connection illustration for the hexahedral element.

In this work, an enhanced explicit Euler integration is employed to solve equation (21) as presented by Huangfu [18]. The enhanced explicit Euler integration is denoted as 
$\dot{\boldsymbol{u}}_{\imath}^{t+1}=\dot{\boldsymbol{u}}^{t}+\Delta t \cdot \frac{\boldsymbol{F}_{i}^{t}}{m_{i}}$

$\boldsymbol{u}_{i}^{t+1}=\boldsymbol{u}_{i}^{t}+\Delta t \cdot \dot{u}_{\imath}{ }^{t+1}$

where $t$ is the current time, $t+1$ is the next time step and $\Delta t$ is the time step.

\section{RESULTS AND DISCUSSION}

\subsection{Stiffness variation in conical spring}

Figure. 4 illustrates the projection view of a conical spring with two coils, which depicts the radius variation. According to equation (2), a bigger radius produces a lower stiffness; thus, when a load is applied to a conical spring, the biggest coil with the lowest stiffness will deflect first. Following that, the deflection will continue indefinitely until all of the coils are fully compressed. Because the radius of the active element reduces, the conical spring becomes stiffer with large deformation, resulting in nonlinear deformation. As shown in Figure. 5, different diameter ratios $\left(D_{1} / D_{2}\right)$ result in different degrees of radius variance. A lower diameter ratio results in a greater degree of radius variation. As a result, the stiffness variation in the conical spring will be higher, resulting in increased nonlinearity.

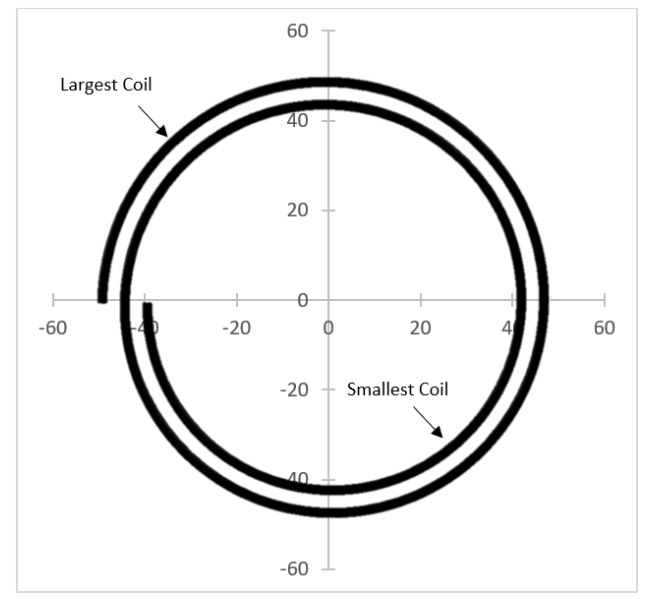

Figure 4: Projection view of the conical spring structure.
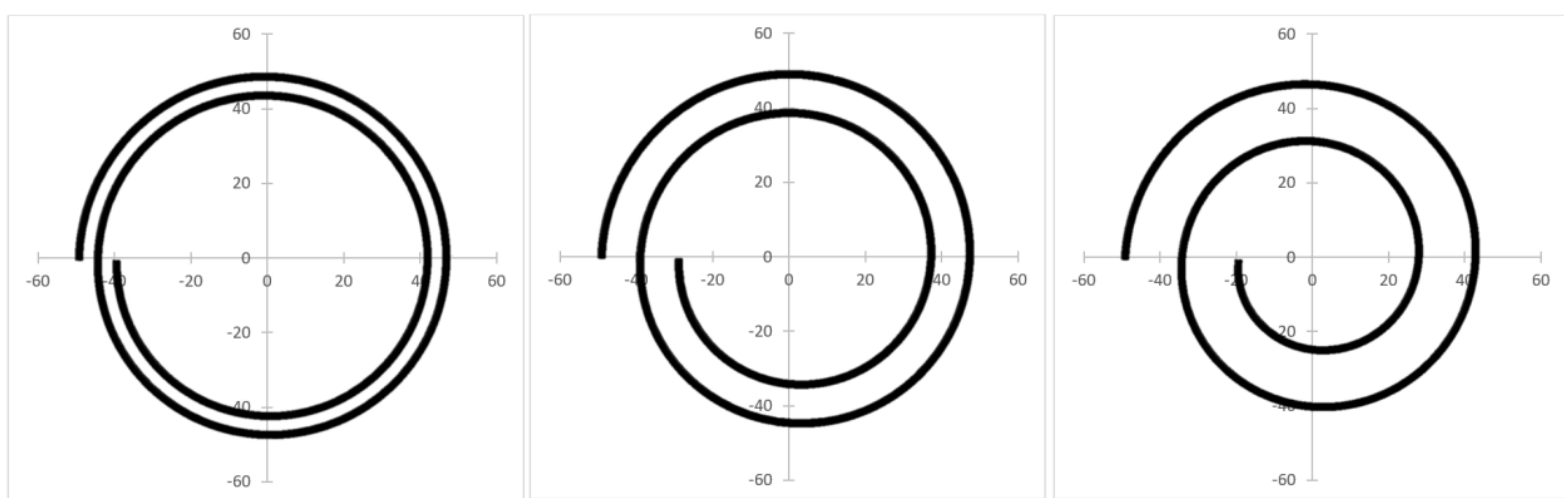

Figure 5: The projection view of conical spring at different ratio of the smallest and the largest coil $\left(D_{1} / D_{2}\right)$. a) Large ratio, b) Medium ratio, and c) Small ratio. 


\subsection{Conical spring deflection behaviors}

This section illustrates how a conical spring may produce several types of deformation. Throughout this experiment, the total number of coils $n_{a}$ and wire diameter $d$ were held constant at 3.0 and $1.0 \mathrm{~mm}$, respectively. The displacement range is set to $90 \mathrm{~mm}$, which corresponds to the maximum elementary deflection $\delta_{e \text { max }}$, and the number of coils used is controlled by the parameter $n$. Thus, the new initial height of the spring $L_{o}$ can be calculated using equation (24).

$L_{o}=\frac{\delta_{e \max } n_{a}}{n}+L_{S}$

The quantity of coil employed within a displacement range might result in a variety of deformation patterns. As demonstrated in Figure. 6, the nonlinearity of a conical spring increases as the number of coils involved within that displacement range grows. The reason for this is that the stiffness variation induced is greater. On the other hand, reducing the stiffness variation by limiting the number of coils involved within that displacement range yields a low nonlinearity. As shown in Figure. 6, when $n=0.5$, the load-displacement curve is very close to a linear behavior.
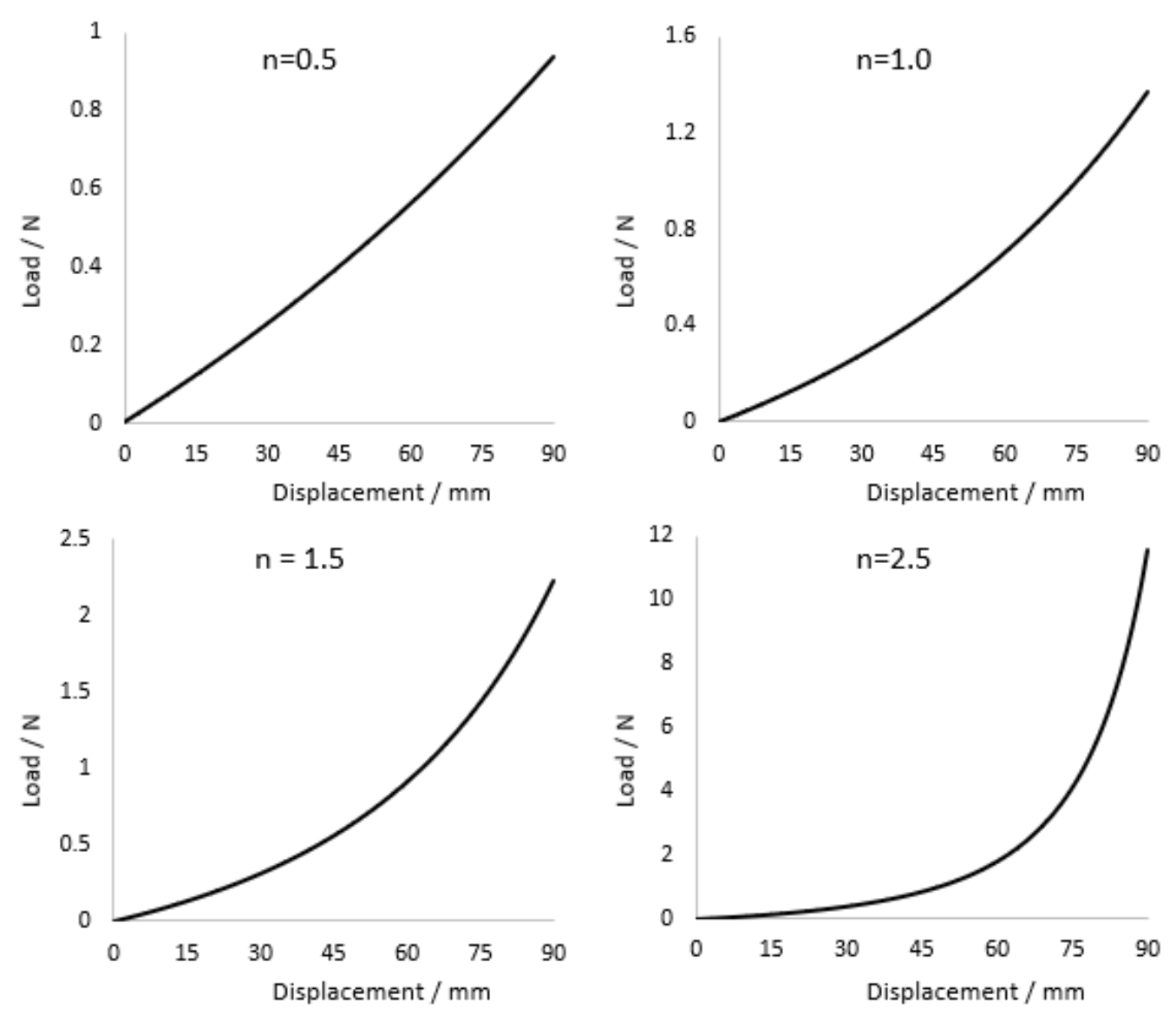

Figure 6: Deformation behaviour of conical spring at different number of coils involved within a given displacement value.

Furthermore, by varying the shear modulus $G$ and wire diameter $d$ in equation (7), the magnitude of spring deflection may be adjusted for a given load P. The two numbers indicate the spring's strength, and they remain constant while the spring deforms. Because the wire diameter $\mathrm{d}$ is fixed in this study, 
the spring strength may be adjusted using the shear modulus value G. When seen in Figure. 7, as G increases, the deflection at a given load $\mathrm{P}$ decreases, but the deformation behavior stays the same.
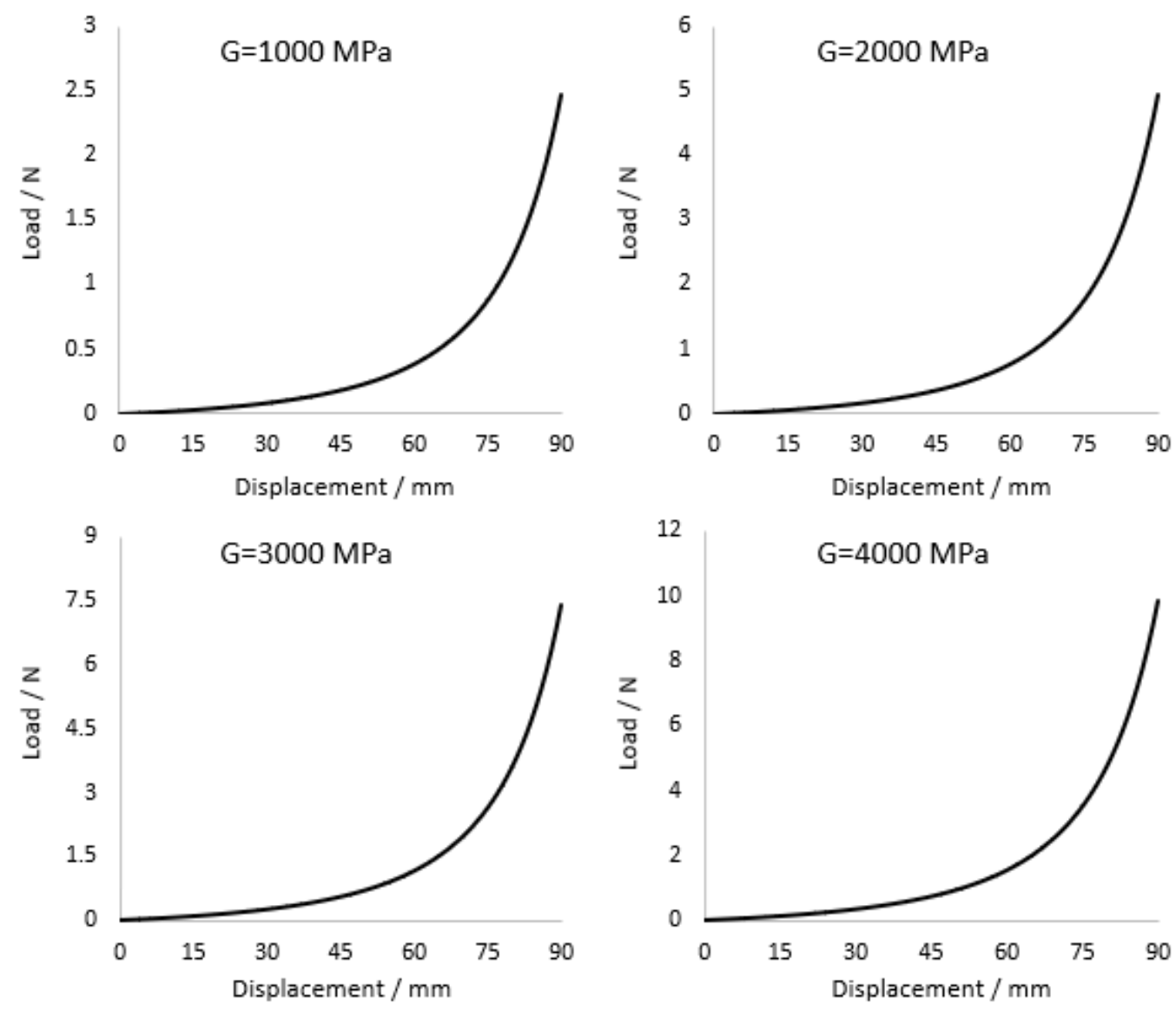

Figure 7: Deformation behaviour of conical spring at different values of shear modulus G.

The results in Figure. 6 and Figure. 7 show that a conical spring may induce various forms of deformation over a wide range of displacements. It is accomplished by modifying the conical spring settings to regulate the stiffness variation. This one-of-a-kind characteristic allows conical springs to represent any sort of deformation behavior.

\subsection{Deformation behaviors}

The ability of the conical spring model to mimic different types of deformation is demonstrated by comparing deformation behavior during compression to that obtained from the literature. Two data sets were obtained directly from Ahmadian et al. [19]. The study examined a variety of soft tissues and determined that there are two main categories of soft tissue deformations that may be distinguished based on the magnitude of the toe region. The toe-region refers to the nonlinear area of soft tissue deformation. The first group includes soft tissues with low nonlinearity in the toe area, such as the human breast and canine kidney. The second group, on the other hand, exhibits more nonlinearity along the toe-region. The findings, as shown in Figure. 8, demonstrate that the conical spring model can replicate multiple types of deformation behavior recorded in the literature. By calibrating the conical spring parameters, which describe the model's flexibility, the different kinds of deformation are duplicated. According to Table 1, the conical spring model's average relative error with respect to the reference data is less than $5 \%$ in both cases. 

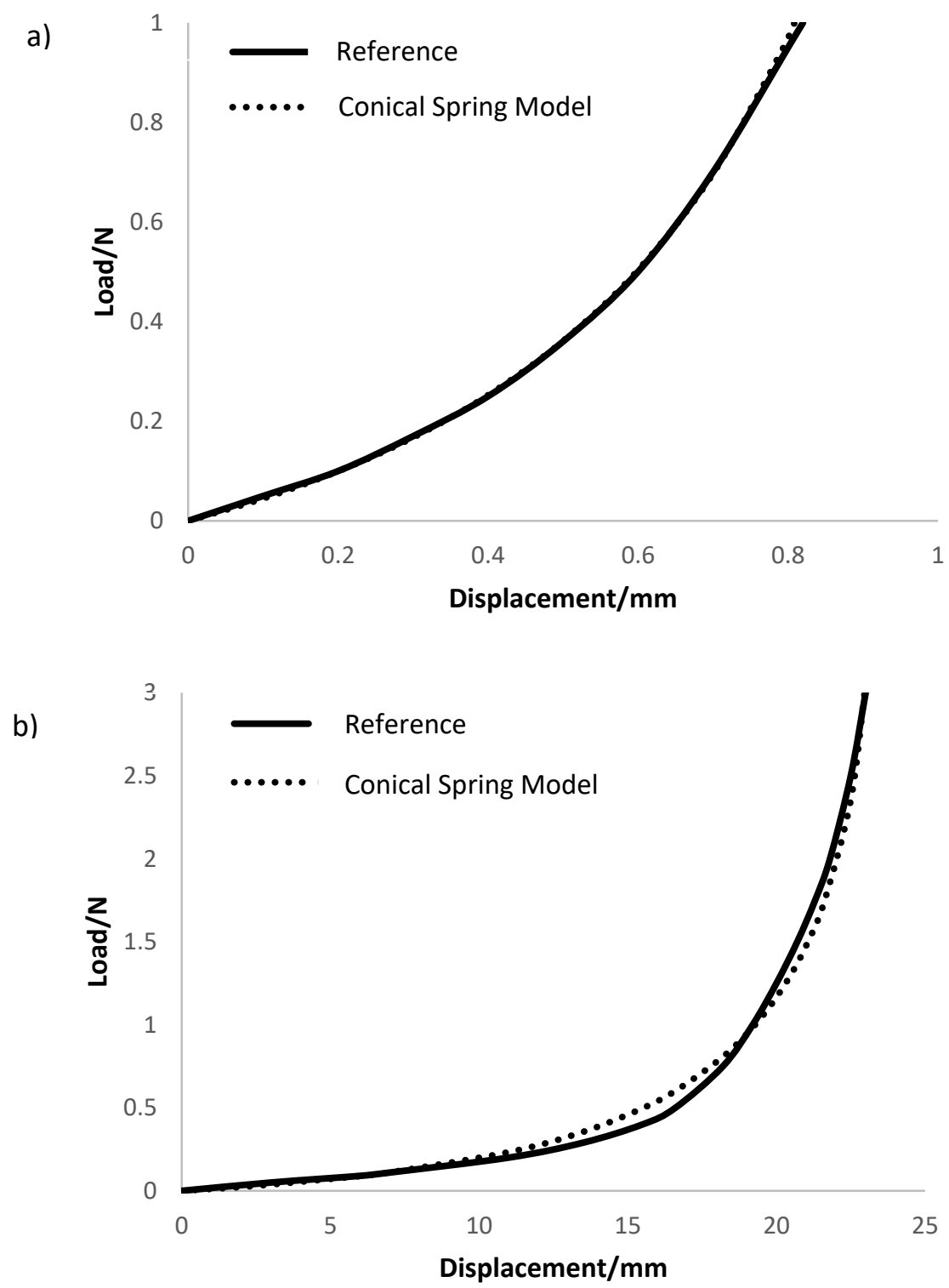

Figure 8: Deformation behaviour of conical spring model in comparison with the reference data of a) Type 1 and b) Type II [19].

Table 1: The relative error of the proposed method in comparison with reference data.

\begin{tabular}{ccc}
\hline Type of deformation & Reference & Average Relative Error $(\%)$ \\
\hline Type I & Figure. 8(a) & 1.89 \\
Type II & Figure. 8(b) & 3.75 \\
\hline
\end{tabular}



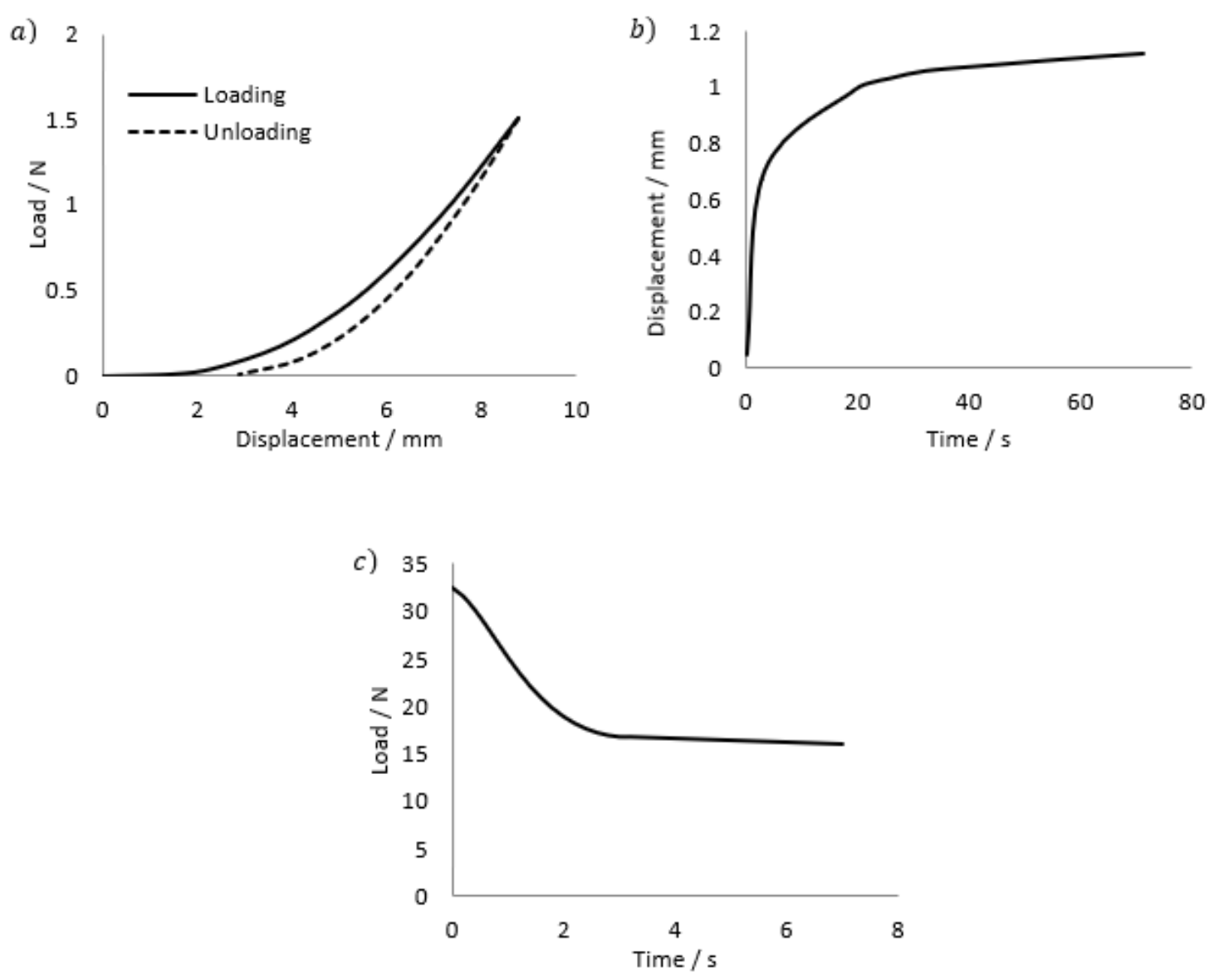

Figure 9: The viscoelastic properties of the conical spring model. a) Hysteresis, b) Creep, and c) Force relaxation.

The existing conical spring model has some limitations, despite its efficacy in simulating different types of deformations. First, the parameters of the conical spring were calibrated using the trial-and-error approach. Although important information was collected from Figure. 6 and Figure. 7, the parameters still do not have a direct relation to soft tissue properties hence the optimization process may be challenging and time consuming for complex data sets.

\subsection{Viscoelastic behavior}

If a model exhibits one of these characteristics, such as hysteresis, creep, or force relaxation, it is considered viscoelastic. According to the hysteresis curve (see Figure. 9(a)), the loading path, represented by the solid line, and the unloading path, indicated by the dotted line, take two separate trajectories. The difference between the pathways represents energy lost throughout the operation, and it is similar to the hysteresis phenomena found in actual tissues [1]. Creeping is the tendency of materials to deform permanently under the presence of continuous pressures, whereas force relaxation is the inclination of materials to show decreases in force when subjected to a steady displacement. At a constant load, the conical spring model continues to deform at a low deformation rate, as seen in Figure. 9(b). The observation is comparable to that reported by Mun et al. [20], which demonstrates the creep feature of soft tissues. Meanwhile, Figure. 9(c) depicts the conical spring model's force relaxation behavior. The response force has a noticeable delay as a result of force relaxation. During an abrupt deformation, the reaction force was seen to have an instantaneous response before progressively declining. The observation is similar to the one reported by Basafa et al. [13]. 
According to the findings of the viscoelastic studies, the conical spring model can replicate the common viscoelastic behaviors of soft tissues. The studies, however, were carried out just to investigate the mechanical properties of the model, with no attempt to imitate any specific viscoelastic material. It is sufficient to modify the damping value as proposed by Sala et al. [21] to achieve different viscoelastic properties.

\subsection{Anisotropic deformation}

The anisotropic property can be incorporated in the conical spring model by allocating different conical spring parameters to distinct spring lattices. Figure. 10 depicts the usage of two different springs (marked by different colors) with the parameters provided in Table 2. When the model is subjected to an external load, it can be seen that the lattices with lower stiffness deform more. The results show that the deformation is direction-dependent, implying that the proposed model can replicate anisotropic characteristics.

Table 2: Details of the conical spring parameters used for the red and white lattices.

\begin{tabular}{ccc}
\hline Parameter & Red lattice & White lattice \\
\hline D1 & 2 & 2 \\
D2 & 8 & 8 \\
Lo & 90 & 90 \\
G & 50 & 50 \\
d & 0.5 & 1.5 \\
$n_{a}$ & 3 & 3 \\
Type & Telescopic & Telescopic \\
Estimated Stiffness & 0.00064 & 0.05149 \\
\hline
\end{tabular}
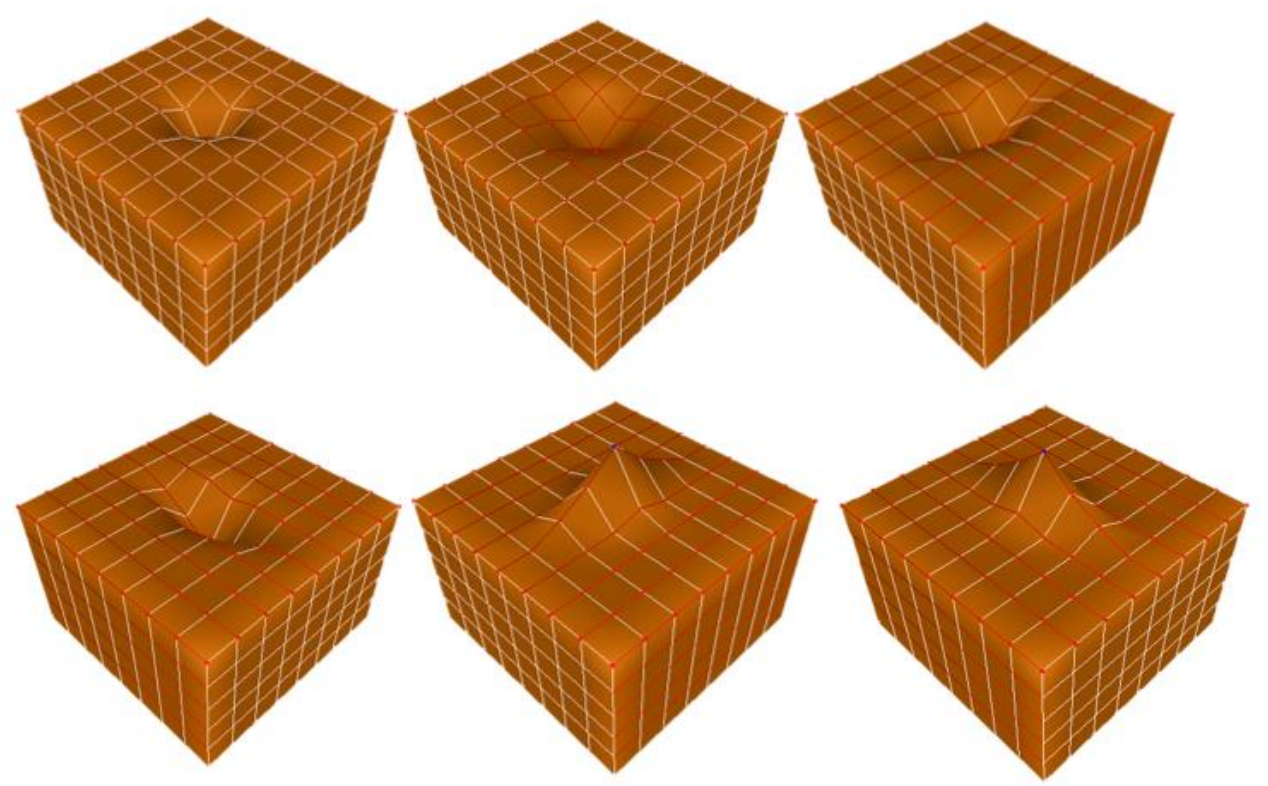

Figure 10: The images of the conical spring model during deformation with anisotropic characteristic is applied. Different lattice colors indicate different spring properties. 


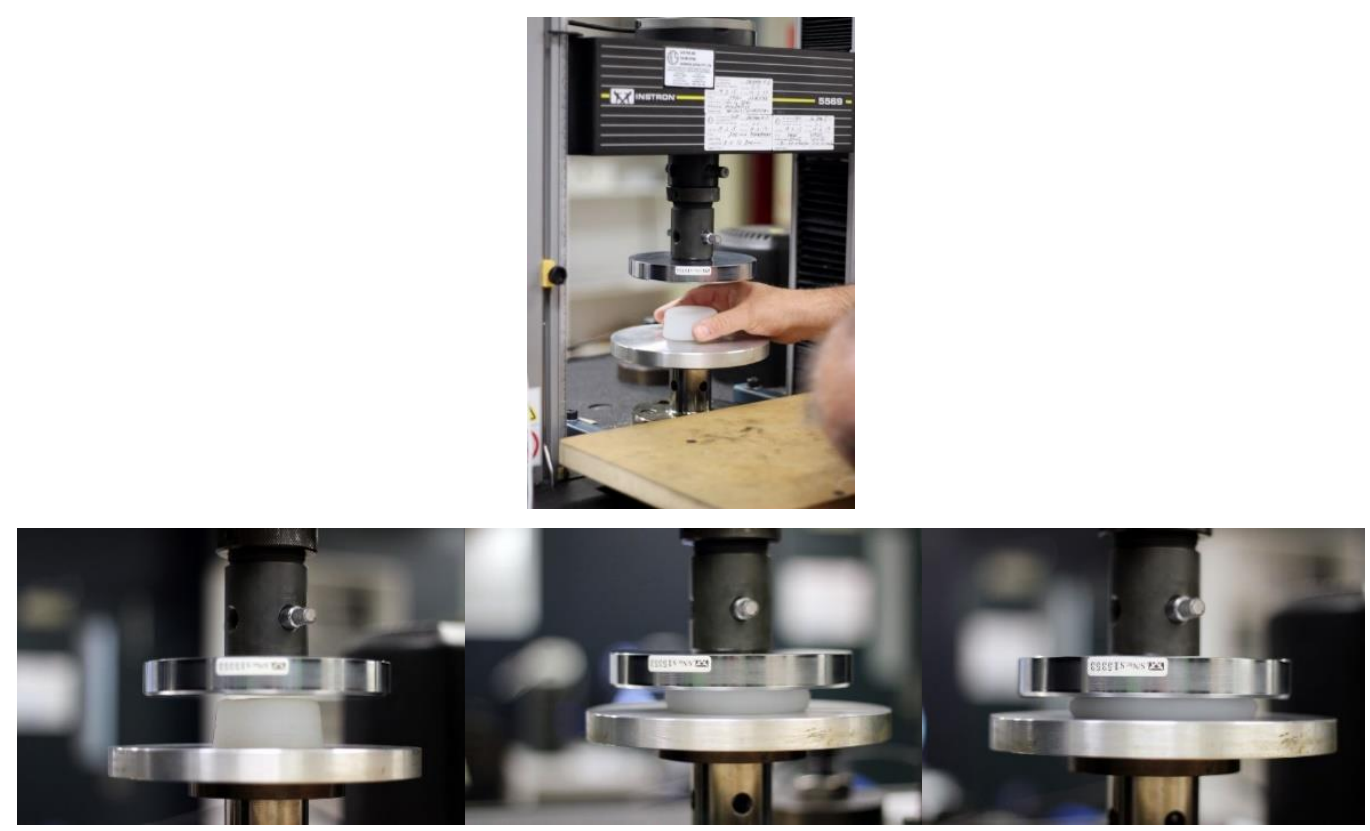

Figure 11: The compression test on the soft tissue phantom (Ecoflex 00-30) using the commercial INSTRON compression machine.

\subsection{Comparison to experimental data}

The deformation behavior of the conical spring model was evaluated with experimental data for further validation. A soft tissue phantom was created using a unique silicone blend of Ecoflex 00-30. According to Spark et al. [22], the Ecoflex 00-30 characteristics are substantially close to those of actual soft tissue, thus it is extensively used as a soft tissue phantom [23]. The specifications of the soft tissue phantom are shown in Table 3.

Table 3: Material specifications of the Silicon Ecoflex 00-30.

\begin{tabular}{|c|c|c|c|c|c|}
\hline $\begin{array}{c}\text { Ecoflex® } \\
00-30\end{array}$ & $\begin{array}{c}\text { Mixed } \\
\text { Viscosity }\end{array}$ & $\begin{array}{l}\text { Specific } \\
\text { Gravity }\end{array}$ & $\begin{array}{l}\text { Specific } \\
\text { Volume }\end{array}$ & Pot Life & Cure Time \\
\hline & $3000 \mathrm{cps}$ & 1.07 & 26.0 & $45 \mathrm{~min}$ & 4 hours \\
\hline Shore Hardness & $\begin{array}{c}\text { Tensile } \\
\text { Strength }\end{array}$ & $100 \%$ Modulus & $\begin{array}{c}\text { Elongation at } \\
\text { Break \% }\end{array}$ & $\begin{array}{l}\text { Die B Tear } \\
\text { Strength }\end{array}$ & $\begin{array}{c}\text { Shrinkage } \\
\text { (in./in.) }\end{array}$ \\
\hline $00-30$ & $200 \mathrm{psi}$ & $10 \mathrm{psi}$ & $900 \%$ & $38 \mathrm{pli}$ & $<.001 \mathrm{in} . / \mathrm{in}$. \\
\hline
\end{tabular}

As illustrated in Figure. 11, the soft tissue phantom was compressed using a commercial INSTRON compression machine. The soft tissue phantom was put on the specimen plate, and compressive stress of $500 \mathrm{~mm} / \mathrm{min}$ was applied (see Figure. 11). For accuracy, the compression test was performed on several specimens. Figure. 12 depicts the test findings, which show that the soft tissue phantom exhibits similar deformation behavior to the first type of soft tissues reported by Ahmadian et al. [19]. 


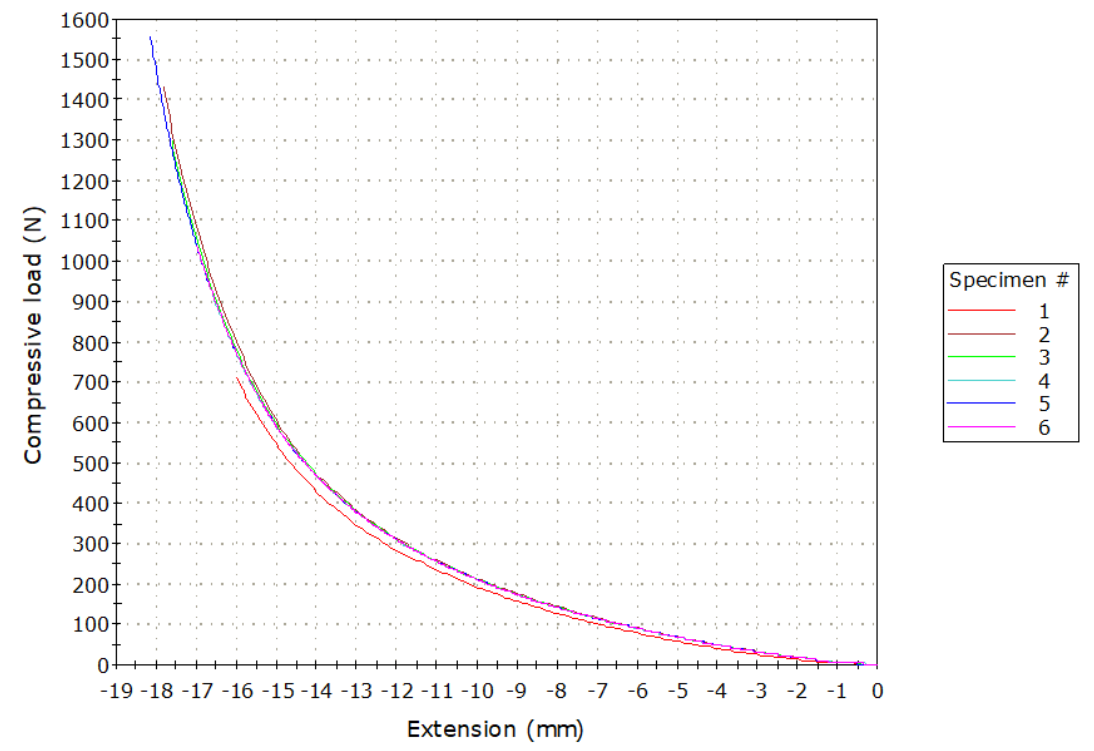

Figure 12: The compression test results on the soft tissue phantom for six specimens taken with the INSTRON compression machine.

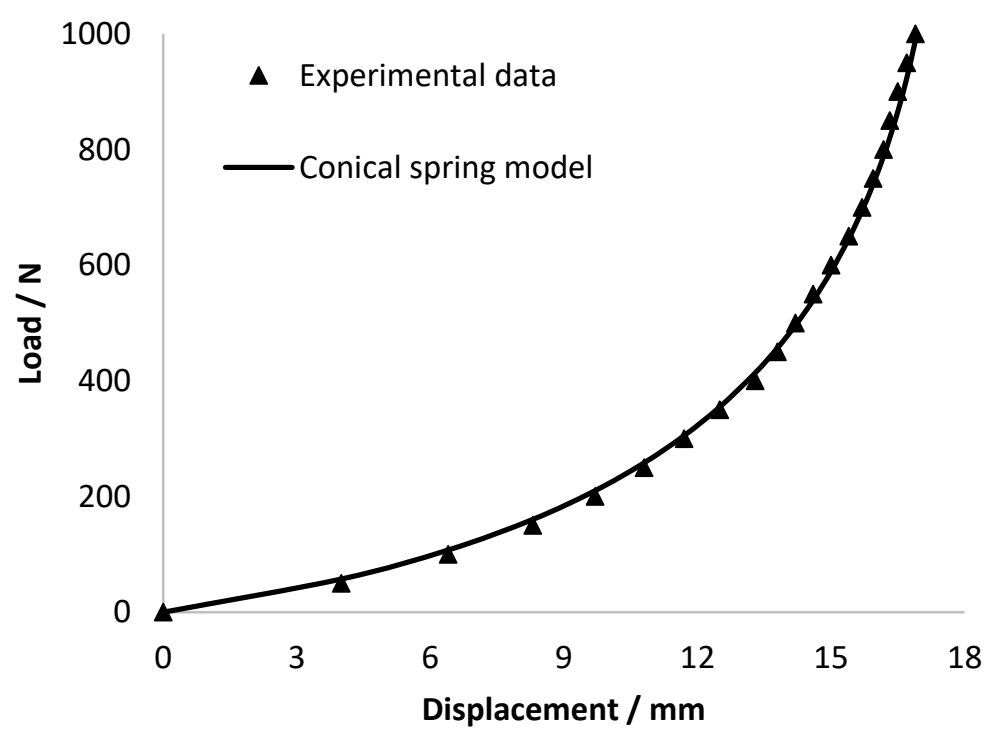

Figure 13: Deformation behaviour of the conical spring model in comparison to the experimental data obtained from the compression test on the soft tissue phantom of Specimen 5.

The deformation behavior of the conical spring model was compared to the deformation behavior of Specimen 5 of the soft tissue phantom. Further calibration was performed on the conical spring parameters to match the deformation behavior of the soft tissue phantom. The calibration process was terminated when the deformation of the conical spring model agreed with the experimental data within $10 \%$ of relative error. As demonstrated in Figure. 13, the conical spring model can simulate the soft tissue phantom's deformation behavior. Although the calibration technique is dependent on trial and error, identical behavior was attained with a relative error of less than $10 \%$. More precise calibration approaches, such as genetic algorithms and simulated annealing, can reduce the percentage of relative inaccuracy even more. 


\subsection{Comparison to traditional MSM}

The deformation behavior and computing time of the conical spring model were compared to those of the traditional MSM model. With 320 nodes, a basic cubical model was created. The traditional MSM model was developed in the same manner as the conical spring model, with the exception that the spring force has been replaced with the linear Hooke's law equation. The conical spring model, as shown in Figure. 14, improves on the classic MSM model by allowing for nonlinear deformation.

a)

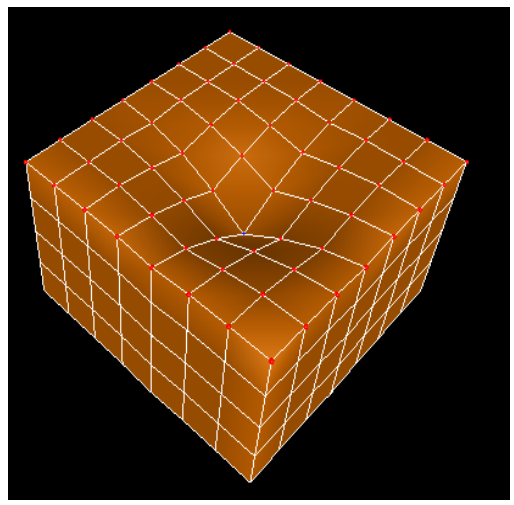

b)

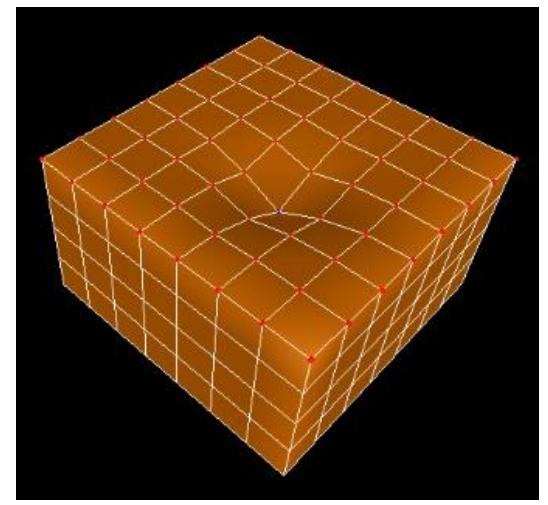

c)

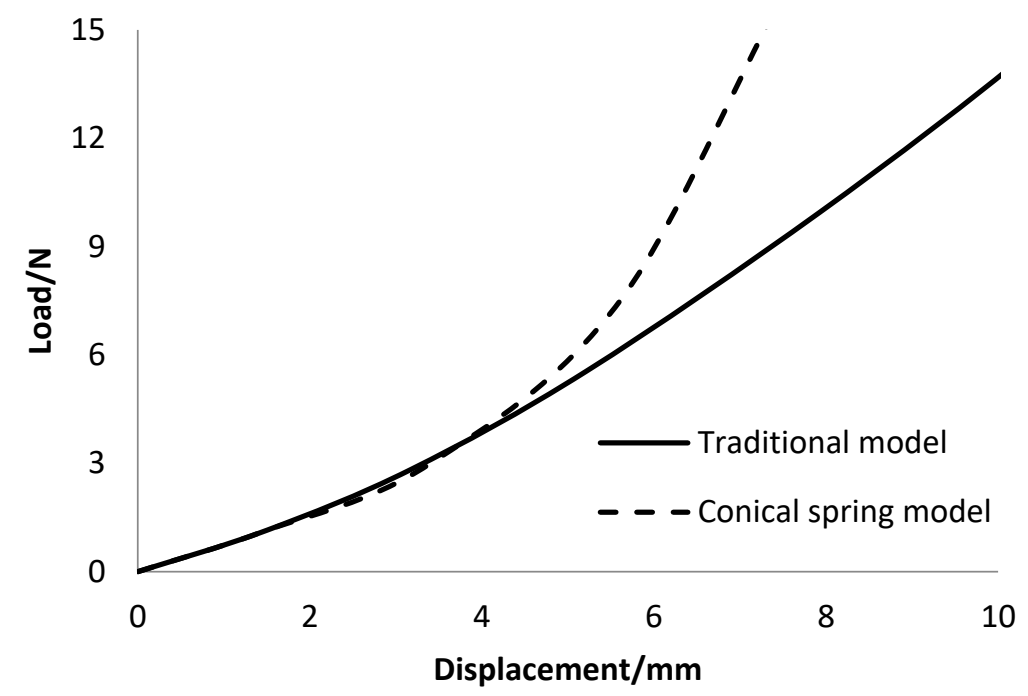

Figure 14: Deformation behaviour of traditional MSM and the conical spring model. a) Deformation image of traditional MSM model, b) Deformation image of conical spring model, and c) Load-displacement relationship of traditional MSM and conical spring model.

However, in terms of computing time, the conical spring model took longer than the traditional approach. The computational time was determined by monitoring the simulation's update time, and the difference is around $7 \mathrm{~ms}$, as shown in Table 4. Although both models were created in the same method, they differ in terms of the mathematical handling of the spring force computation. Because the basic linear Hooke's law equation was employed, the traditional MSM model has an advantage. However, it is confined to linear deformation, which does not represent the general behavior of soft tissues. Nevertheless, even though the spring force for the conical spring model is more complicated, a more accurate simulation can be generated. As a result, the $7 \mathrm{~ms}$ difference might be deemed minor in comparison to the capacity to achieve greater precision. 
Table 4: Update time of the conical spring model and traditional MSM.

\begin{tabular}{ccc}
\hline MSMs & Number of nodes & Update time (s) \\
\hline Conical spring model & 320 & 0.090 \\
Traditional MSM & 320 & 0.083 \\
\hline
\end{tabular}

\subsection{Interactive feedback}

The SOFA framework software was used to assess the interactive capability of the conical spring model employed in human organs. SOFA is an open-source framework that allows independently developed algorithms to communicate within the context of a shared simulation [24]. This tool allows for realtime interaction with the model numbers that are accessible. Changes were made to the header and inline files to implement the conical spring methodology in the SOFA framework. Using the conical spring equation, the files relevant to the Spring-Force were explicitly altered. Figure. 15 shows the deformations of the modified liver model, which has 507 nodes. The results demonstrate that the conical spring model can be operated at a real-time interactive phase, which is essential for haptic feedback.
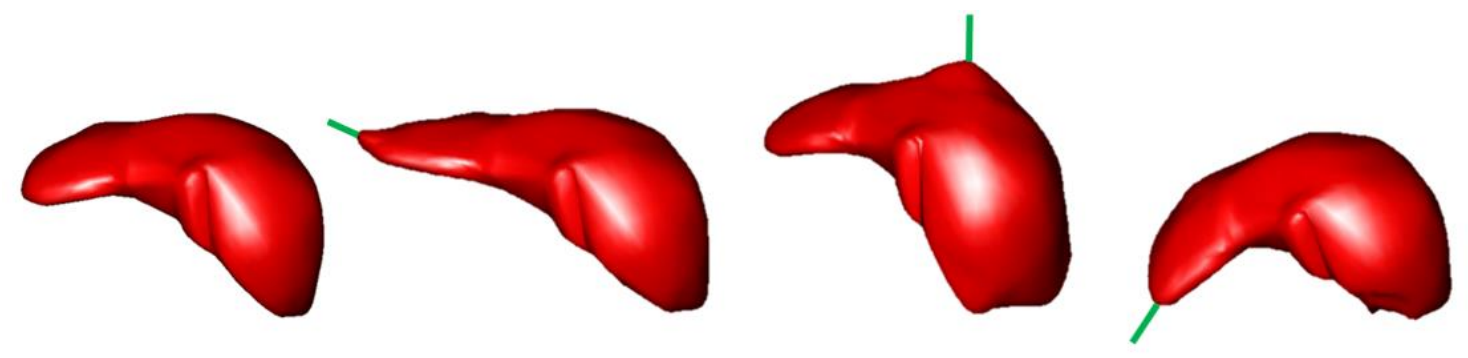

Figure 15: Implementation of the proposed method in SOFA open source. Image of the original liver model followed by deformed model.

\section{CONCLUSIONS}

The goal of this research is to develop a new framework for simulating soft tissue deformation. Instead of oversimplifying soft tissue deformation as nonlinear or linear, this work indicates that the deformation behavior is determined by a combination of linear and nonlinear deformation phases. It was achieved using the conical spring methodology in which the unique conical structure generates stiffness variation within the conical spring model. The stiffness variation can be regulated using the conical parameters to match any deformation behavior. The results show that the conical spring model has the flexibility in modeling different types of soft tissue deformation with high precision, thus eliminates the slight computational time disadvantage when compared to the traditional MSM model. Additionally, the conical spring model is easily adaptable to show the general viscoelastic and anisotropic mechanical properties of soft tissues.

\section{ACKNOWLEDGEMENT}

The authors would like to thank to the RMIT University and Universiti Malaysia Pahang for financial assistance under Research Grant project No. RDU1803146.

\section{REFERENCES}

[1] Fung, Yuan-Cheng. Biomechanics: Mechanical Properties of Living Tissues. 3rd ed., Springer, 1993. 
[2] Holzapfel, G.A. Biomechanics of soft tissue. The handbook of materials behavior models, 3, 1049-1063, 2001.

[3] Pierorazio, P.M. and Allaf, M.E. Minimally invasive surgical training: challenges and solutions. In Urologic Oncology: Seminars and Original Investigations, 27(2), 208-213, 2009.

[4] Schreuder, H.W., Wolswijk, R., Zweemer, R.P., Schijven, M.P. and Verheijen, R.H. Training and learning robotic surgery, time for a more structured approach: a systematic review. BJOG: An International Journal of Obstetrics \& Gynaecology, 119(2), 137-149, 2012.

[5] Cooper, L., and Maddock, S. Preventing collapse within mass-spring-damper models of deformable objects. 5th International Conference Centre Europe, Computer Graphic Visual, 2(1), 196-204, 1997.

[6] Teschner, M., Girod, S. and Girod, B. Direct Computation of Nonlinear Soft-Tissue Deformation. Vis. Model. Vis., 22-24, 2000.

[7] Luo, Q. and Xiao, J. Contact and deformation modeling for interactive environments. IEEE Transactions on Robotics, 23(3), 416-430, 2007.

[8] Cui, T., Song, A. and Wu, J. Simulation of a mass-spring model for global deformation. Frontiers of Electrical and Electronic Engineering in China, 4(1), 78-82, 2009.

[9] San-Vicente, G., Aguinaga, I. and Celigueta, J.T. Cubical mass-spring model design based on a tensile deformation test and nonlinear material model. IEEE Transactions on Visualization and Computer Graphics, 18(2), 228-241, 2012.

[10] Shah, R. and Gupta, A. Non-linear cubic spring-mesh model for simulating biological tissues. Bio-Medical Engineering Science, 51(Supplement), U-12, 2013.

[11] Choi K-S, Sun H, Heng P-A. Interactive deformation of soft tissues with haptic feedback for medical learning. IEEE Transactions on Information Technology in Biomedicine, 7(4), 358363, 2003.

[12] Choi, K.S., Sun, H., Heng, P.A. and Zou, J. Deformable simulation using force propagation model with finite element optimization. Computers \& Graphics, 28(4), 559-568, 2004.

[13] Basafa, E. and Farahmand, F. Real-time simulation of the nonlinear visco-elastic deformations of soft tissues. International journal of computer assisted radiology and surgery, 6(3), 297-307, 2011.

[14] Keeve, E., Girod, S., Kikinis, R. and Girod, B. Deformable modeling of facial tissue for craniofacial surgery simulation. Computer aided surgery, 3(5), 228-238, 1998.

[15] García, M., Gómez, M., Ruíz, Ó. and Boulanger, P. Spring-particle model for hyperelastic cloth. In Canadian Congress of Applied Mechanics, CANCAM, 2005.

[16] Wahl, A.M. Mechanical springs. Penton Publishing Company, 1944.

[17] Paredes, M., and Rodriguez, E. Optimal design of conical springs. Engineering with computers, 25(2), 147-154, 2009.

[18] Huangfu, Z. An Improved Mass-Spring Model for Simulation of Soft Tissue Deformation. Journal of Information and Computational Science, 10(17), 5551-5558, 2013.

[19] Ahmadian, M.T. and Nikooyan, A.A. Modeling and prediction of soft tissue directional stiffness using in-vitro force-displacement data. Int. J. Sci. Res, 16, 385-389, 2006.

[20] Mun, P., Zhong, Y., Shirinzadeh, B., Smith, J. and Gu, C. An improved mass-spring model for soft tissue deformation with haptic feedback. In ICMT 2011, 1-6, 2011.

[21] Sala, A., Turini, G., Ferrari, M., Mosca, F. and Ferrari, V. Integration of biomechanical parameters in tetrahedral mass-spring models for virtual surgery simulation. In Engineering in Medicine and Biology Society, EMBC, 2011 Annual International Conference of the IEEE, 4550-4554, 2011.

[22] Sparks, J. L., Vavalle, N. A., Kasting, K. E., Long, B., Tanaka, M. L., Sanger, P. A., Schnell, K., and Conner-Kerr, T. A. Use of Silicone Materials to Simulate Tissue Biomechanics as Related to Deep Tissue Injury. Advances in Skin \& Wound Care, 28(2), 59-68, 2015. 
[23] Boonvisut, P. and Cavusoglu, M. Estimation of Soft Tissue Mechanical Parameters from Robotic Manipulation Data. IEEE/ASME Transactions on Mechatronics, 18(5), 1602-1611, 2013.

[24] Allard, J., Cotin, S., Faure, F., Bensoussan, P.J., Poyer, F., Duriez, C., Delingette, H. and Grisoni, L. Sofa-an open source framework for medical simulation. In MMVR 15-Medicine Meets Virtual Reality, 125(1), 13-18, 2007. 